\title{
Parental education and adult health outcomes: a cohort study examining disease-specific effects of education levels using Swedish nationwide registries across two generations
}

\author{
Hendrik Koffijberg \\ Julius Center for Health Sciences and Primary Care, University Medical Center Utrecht, Utrecht \\ h.koffijberg@umcutrecht.nl \\ Johanna Adami \\ Clinical epidemiology unit, Department of Medicine Solna, Karolinska Institutet, Stockholm \\ Erik Buskens \\ Department of Epidemiology, University Medical Center Groningen, University of Groningen, Groningen \\ Mårten Palme \\ Department of Economics, Stockholm University, Stockholm
}

(Received August 2011 Revised April 2012)

\section{Abstract}

It is well known that children with less educated parents have inferior health status in later life. There are two competing hypotheses explaining the association found: the pathway hypothesis - suggesting that children from low educated households are more likely to obtain less education themselves, which, in turn, is associated with inferior health outcomes - and the life course hypothesis - suggesting that living conditions during childhood, as such affected by parental education level, is important for the formation of adult health status. We obtained data from National Swedish registries comprising health outcomes of individuals born between 1940 and 1949, and the Swedish Multi-generation Register. We assessed the differences in risk of hospital admission for individuals with low and high parental education as well as low and high own education. We found that for higher educated individuals, high parental education is associated with even better health outcomes: having a high versus low educated mother or high versus low educated father was associated with an overall decrease in the risk (hazard rate) of hospital admission by 5\% (95\% $\mathrm{Cl} 0.91-0.98)$ and $3 \%$ (95\% $\mathrm{Cl} 0.95-0.99)$, respectively. This indicates that children from a relatively disadvantaged background, signaled by lower parental educational attainment, are more likely to continue accumulating risk throughout life. Even if they have higher qualifications they may still have a greater accumulation of risk, compared with other highly qualified children from a less disadvantaged background. We found that this effect is primarily attributed to circulatory diseases, and would appear to support the life course hypothesis. We conclude that parental education and ensuing early childhood or even fetal living conditions have a persistent effect on adult health.

Keywords: inter-generational; education; life course study; pathway hypothesis 


\section{Introduction}

It has been established that children with less educated parents have inferior health status in their childhood and later in life compared to children with high educated parents (Case, Lubotsky \& Paxson, 2002; Gakidou et al., 2010). Parental education may influence adult health only indirectly, i.e. as a result of the fact that children with low educated parents are themselves likely to obtain lower final educational levels, in turn associated with inferior health outcomes. This indirect effect is called the pathway hypothesis. (Marmot et al., 2001). Conversely, the inferior health may also be explained through a lasting, or accumulated, effect of inferior living conditions during childhood and adolescence. This direct effect is called the life course hypothesis (Kuh et al., 2003). The life course hypothesis can be further divided into (i) the accumulation, (ii) the critical period and (iii) social mobility hypotheses (Mishra et al., 2009).

Although evidence in support of the life course hypothesis exists, this evidence is mainly based on observations of child health, or adolescent health (Kuh et al., 2003). In studies on health outcomes at relatively young ages, a higher prevalence of diseases among children in families with lower socio-economic status was found (Case et al., 2002). Also, disease severity was worse among these families. Given that children born in families with low socio-economic position (SEP) have inferior health, it is then assumed that health problems at a young age are also related to poor health outcomes later in life. However, directly linking parental education to actual health outcomes later in life remains difficult, due to the long latency period in both cardiovascular disease and cancer (Gluckman et al., 2008).

In this study we evaluated the relation between parental education and subsequent health in later life as actually observed, i.e. hospital admission and death occurring in individuals older than 50 years. To this end, we used extensive registry data on a large population-based cohort with long term follow-up and information about potential confounders. Our data includes International Classification of Diseases (ICD) 9 or 10 codes for all hospital admissions in Sweden for the period 19872006 and data from the cause of death register. Thus, the usual extrapolation from child health to adult health could be avoided.
We studied the interaction between own education and paternal as well as maternal education on the health outcomes. This framework allowed us to test the pathway against the life course hypotheses. The life course hypothesis would predict an independent effect of parental education conditional on own education. We also discussed to what extent the results may be attributed to each of the three life course hypotheses mentioned above.

Since the available data did not allow us to identify the causal effect of education on health outcomes - i.e. we were unable to separate out the effect of education itself from all possible confounding factors - we limited the study to view education as a marker of socio-economic position.

\section{Method}

\section{Data selection}

In our study, we included all individuals born in Sweden between 1940 and 1949 and obtained the identities of these individuals from the population census. Information on date and region of birth as well as gender for the index persons and their parents, was collected from the population census (Statistics Sweden, 2009a). The linkage between the two generations was achieved through the Swedish Multi-generation Register (Statistics Sweden, 2009b).

Health

Data on all hospital admissions between 1987 and 2005 were collected from the Swedish National Patient Register (Socialstyrelsen, 2009a). This register contains administrative information such as date of admission, number of days in hospital care, as well as discharge diagnoses classified according to ICD 9 or ICD 10 codes. This data did not include out-patient visits and day surgery. Data on the occurrence of co-morbidities was limited and likely to be less accurate than data on the main disease of interest. Therefore, we did not use co-morbidity data in our analysis.

Information on the cause of death, for deceased index persons, was obtained from the National Cause of Death Registry for the period 1987-2006 (ICD 9 and ICD 10 codes) (Socialstyrelsen, 2009b). Data on all hospital admissions due to cancer were obtained from the Swedish Cancer Registry (Socialstyrelsen, 2009c). This register covers the whole population of Sweden and comprises all cancer diagnoses recorded, among others, as ICD 7 
codes. The completeness of the registries has previously been shown to be very high (Barlow et al., 2009; Socialstyrelsen, 2009a; Socialstyrelsen, 2009b; Socialstyrelsen, 2009c).

\section{Education}

Data on highest educational level for the index persons was collected from the 1990 records in the Swedish Register of Education (Statistics Sweden, 2004). For the parents, the corresponding information was obtained from the 1970 census (Statistics Sweden, 2009a). The original education levels were re-coded into low education level, i.e. compulsory education up to vocational schooling, and high education level, i.e. upper secondary education up to doctoral education, for both index persons and parents. This categorization was required as the use of multiple education levels would result in a too large number of combinations of education of the index person, the father, and the mother. Moreover, only very few of the parents attained high education levels, which would result in many combinations with very few index persons and parents if more than two education levels were used.

\section{Income and immigration}

Data on taxable income from labour in 1990 was obtained for the index persons from the National Tax Register (Statistics Sweden, 2009c). The population census was used to collect information on migration status.

\section{Definition of the cohort}

In order to have accumulated a substantial medical history, the selected index persons should be as old as possible, e.g. around the age of 70 in 1990. However, parental education levels were only available for parents born from 1912 onwards. Therefore, we selected individuals born in the period 1940-1949, who had parents born from 1912 onwards, as index persons. Subsequently we assessed the medical history of the index persons starting from the age of 50 years. As a result, any disease histories resulting in death before the age of 50 were not taken into account. For each index person we retrieved all relevant hospital admissions and, if the index person had died, the cause of death. The risk of hospital admission related to cancer was ascertained twice, once using the
Swedish National Patient Register and once using the Swedish Cancer Registry.

\section{Statistical analyses}

First, we estimated differences in incidence rates of hospital admission for index persons with low and with high education. Second, we estimated incidence rate ratios for the effects of high versus low maternal education level, and high versus low paternal education level, separately for index persons with low and high education level. Homogeneity of these groups of index persons was assessed with the Mantel-Haenszel test (Kirkwood and Sterne, 2003). Finally, we used Cox proportional hazard competing risk models (Kirkwood and Sterne, 2003). The dependent variable was time to the event under study, with the event defined as the primary diagnosis for hospital admission or death of the index person. For hospital admission, time to first hospital visit beyond the age of 50 years, caused by the indicated diagnosis was used. For mortality, the time to death by diagnosis group for index persons dying after the age of 50 years was used. For the analysis of mortality this means that individuals dying from another diagnosis than the considered type are treated as right censored at the date of death. Variables in the Cox model explaining the time to event were: education of the mother (high/low), education of the father (high/low), income of the index person (tertiles), sex, year of birth of the index person (1940/41, $1942 / 43, \ldots, 1948 / 49)$, period of birth of the mother $(1912 / 21,1922 / 31)$, period of birth of the father (1912/21, 1922/31), adoption (yes/no), and region of birth in Sweden (northern, central, southern). Index persons with low and high education were analyzed separately. All analyses were performed with STATA 9.2 SE (StataCorp, Texas, USA).

\section{Results}

Figure 1 shows the flowchart for the data retrieval and selection process. Data was available on all relevant variables for 458,966 index persons and their parents after data selection. A large number of index persons had to be excluded, due to missing data on parental education levels. This selection accounted for $97 \%$ of all excluded index persons, and was mainly caused by one parent or both parents dying before the 1970 census in which data on education levels was collected. 
Figure 1. Flowchart of the data retrieval and selection process

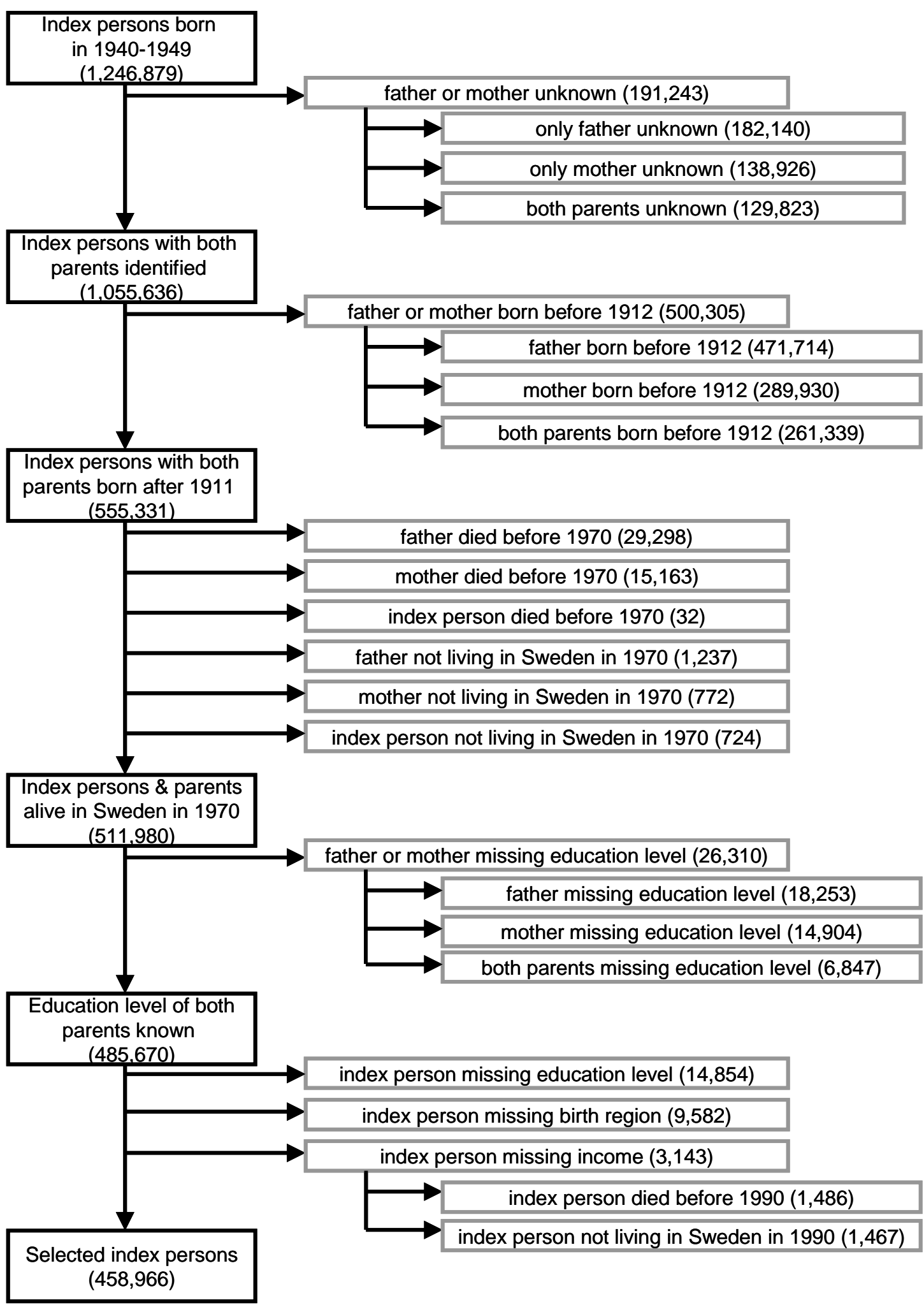

Notes. The majority of missing education levels for parents or index persons may be caused by death of the individuals prior to 1952. Education levels were determined in the 1970 census which was mandatory for all Swedish residents below the age of 65 , i.e. born after 1911. However, death before 1970 cannot always be ascertained since the cause of death register was started on 1/1/1952. Parents and index persons dying before 1952 have no date of death, and are therefore assumed to be still alive in 1970 and 1990. These individuals will cause missing education levels and missing income data. 


\section{Table 1. Characteristics of the selected index persons and their parents}

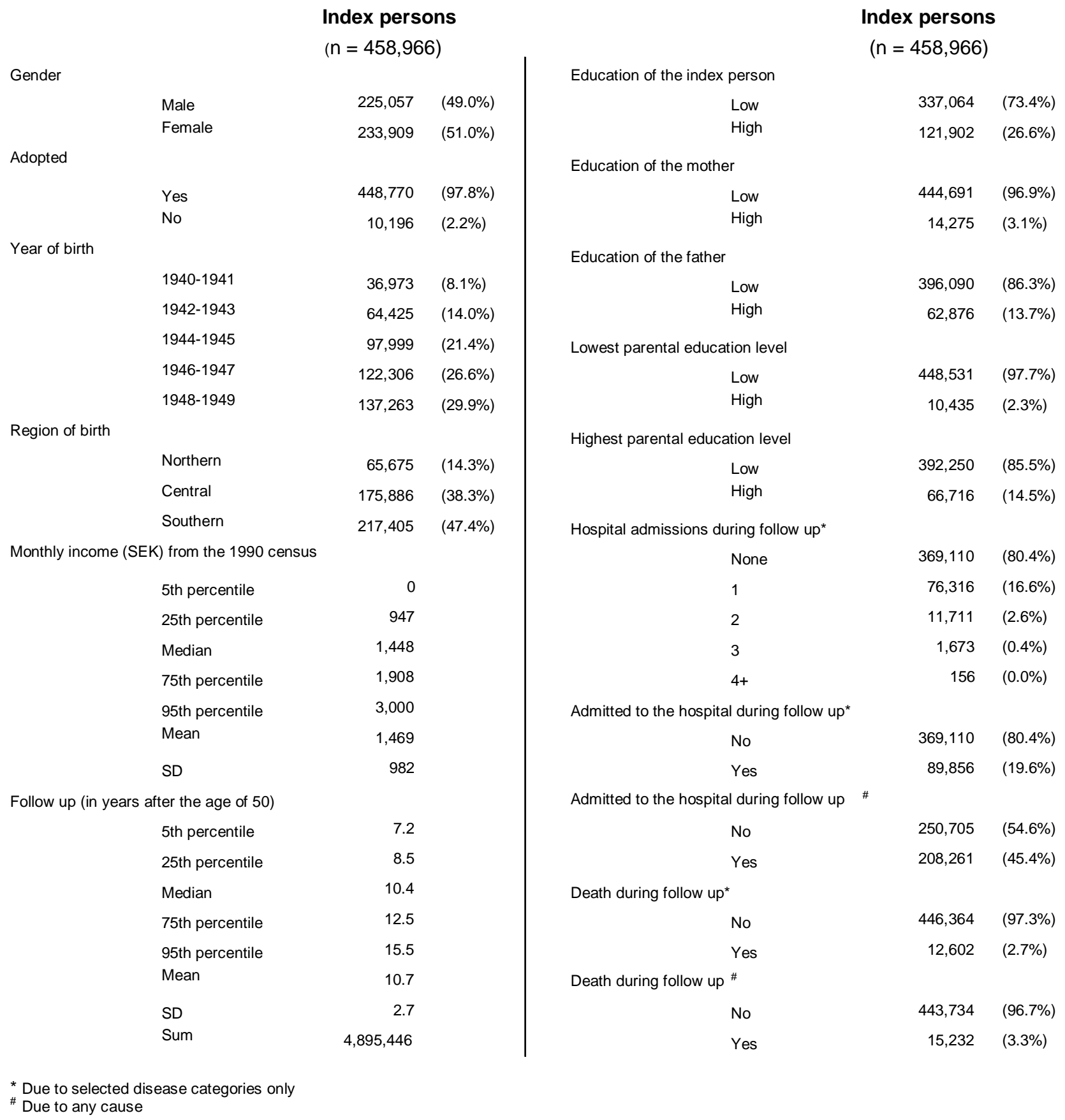

Notes. Low education level was defined as compulsory education up to vocational education. High education level was defined as upper secondary education up to doctoral education.

Table 1 shows descriptive statistics of the final dataset. In general, education of the father was higher than that of the mother. Furthermore, education of the index-persons was in general higher than parental education levels. Because the average age at the end of follow up was around 61 years, more than half of the index persons (54.6\%) had not been hospitalized, and only $3.3 \%$ had died during follow up. In total 4.9 million years of follow up were available. The effect of education of the index persons on the risk of hospital admission is shown in Table 2. In this table, all rate differences are positive; indicating that a low education level of the index persons increased their risk of hospital admission, for all disease categories considered. 
Table 2. Differences in incidence rates of hospital admission for low and high educated index persons

\begin{tabular}{|c|c|c|c|c|c|c|c|c|}
\hline & \multicolumn{2}{|c|}{$\begin{array}{l}\text { Index persons with } \\
\text { low education } \\
(n=337,064)\end{array}$} & \multicolumn{2}{|c|}{$\begin{array}{l}\text { Index persons with } \\
\text { high education } \\
(n=121,902)\end{array}$} & \multicolumn{2}{|c|}{$\begin{array}{c}\text { Rate difference } \\
\text { low vs high education } \\
(x 10,000)\end{array}$} & \multicolumn{2}{|c|}{$\begin{array}{c}\text { Rate ratio } \\
\text { low vs high education }\end{array}$} \\
\hline & Events & PY & Events & PY & Estimate & $95 \% \mathrm{Cl}$ & Estimate & $95 \% \mathrm{Cl}$ \\
\hline All hospital admissions combined & 159,769 & $2,608,416$ & 48,460 & 993,770 & 125 & $(120-130)$ & 1.26 & $(1.24-1.27)$ \\
\hline Cancer [140-208 / C00-C97] & 19,911 & $3,543,764$ & 6,447 & $1,258,696$ & 5 & $(3-6)$ & 1.10 & $(1.07-1.13)$ \\
\hline Respiratory diseases [460-519 / J00-J99] & 13,712 & $3,541,744$ & 3,193 & $1,265,559$ & 13 & $(12-15)$ & 1.53 & $(1.48-1.60)$ \\
\hline Diabetes [250 / E10-E14] & 3,912 & $3,591,958$ & 781 & $1,277,846$ & 5 & $(4-5)$ & 1.78 & $(1.65-1.93)$ \\
\hline Chronic liver disease [571 / K70,K73-K74] & 1,093 & $3,609,462$ & 228 & $1,281,152$ & 1 & $(1-2)$ & 1.70 & $(1.47-1.97)$ \\
\hline Mental disorders $[303,305$ / F10] & 5,987 & $3,577,571$ & 1,128 & $1,275,655$ & 8 & $(7-9)$ & 1.89 & $(1.78-2.02)$ \\
\hline Circulatory diseases [390-459 / 100-199] & 37,986 & $3,415,613$ & 11,005 & $1,226,142$ & 21 & $(19-23)$ & 1.24 & $(1.21-1.27)$ \\
\hline - Cerebrovascular disease [430-438 / I60-169] & 7,409 & $3,580,231$ & 2,013 & $1,273,204$ & 5 & $(4-6)$ & 1.31 & $(1.25-1.38)$ \\
\hline - Ischemic heart disease [410-414 / I20-I25] & 15,667 & $3,532,206$ & 4,092 & $1,261,839$ & 12 & $(11-13)$ & 1.37 & $(1.32-1.42)$ \\
\hline - Cerebrovascular or ischemic heart disease & 22,204 & $3,496,604$ & 5,915 & $1,252,279$ & 16 & $(15-18)$ & 1.34 & $(1.31-1.38)$ \\
\hline - Other circulatory diseases & 15,782 & $3,532,380$ & 5,090 & $1,255,937$ & 4 & $(3-5)$ & 1.10 & $(1.07-1.14)$ \\
\hline
\end{tabular}

Notes. The observed number of hospital admissions and person-years for index persons with low and high education level. For each disease category the rate difference and rate ratio for low versus high educated index persons is given, along with the corresponding 95\% confidence intervals.

$P Y=$ person-years; $\mathrm{Cl}=$ confidence interval. 
To investigate whether the effects of maternal and paternal education level are similar in index persons with low and with high education, crude effects were estimated, as ratios of incidence rates, and are shown in Tables 3A-B. From these tables we see that, in general, high education of the mother and high education of the father reduced the risk of hospital admission, in both index persons with low and with high education themselves. However, for many disease categories, this reduction is more pronounced in individuals with high education level. Also, high maternal education appeared to be slightly more beneficial than high paternal education in most disease categories. The Mantel-Haenzel test indicated that the two subgroups of index persons were not homogeneous for several disease categories. In our subsequent, adjusted analyses, based on the Cox model, we therefore stratified by education level of the index persons.

\section{Table 3. Crude effects of parental education level for index persons with low and high education}

[A]

\section{High vs low education of the mother}

\begin{abstract}
All hospital admissions combined
Cancer [140-208 / C00-C97]

Respiratory diseases [460-519 / J00-J99]

Diabetes [250 / E10-E14]

Chronic liver disease [571 / K70,K73-K74]

Mental disorders [303,305 / F10]

Circulatory diseases [390-459 / 100-199]

- Cerebrovascular disease [430-438 / 160-169]

- Ischemic heart disease [410-414 / 120-125]

- Cerebrovascular or ischemic heart disease

- Other circulatory diseases
\end{abstract}

[B]

\section{High vs low education of the father}

Index persons with
low education
$(\mathrm{n}=337,064)$
IRR $\quad 95 \% \mathrm{CI}$

$\begin{array}{ll}0.92 & (0.87-0.96) \\ 0.99 & (0.85-1.13) \\ 0.79 & (0.65-0.95) \\ 0.85 & (0.59-1.20) \\ 0.72 & (0.31-1.42) \\ 1.06 & (0.81-1.35) \\ 0.84 & (0.75-0.93) \\ 1.01 & (0.80-1.27) \\ 0.71 & (0.58-0.85) \\ 0.80 & (0.68-0.92) \\ 0.91 & (0.77-1.07)\end{array}$

Index persons with low education ( $n=337,064$ )

IRR 95\% Cl

$\begin{array}{ll}0.97 & (0.96-0.99) \\ 1.06 & (1.01-1.12) \\ 0.96 & (0.90-1.02) \\ 0.92 & (0.81-1.03) \\ 1.02 & (0.81-1.28) \\ 1.05 & (0.96-1.16) \\ 0.89 & (0.86-0.93) \\ 0.93 & (0.85-1.02) \\ 0.82 & (0.77-0.88) \\ 0.86 & (0.82-0.91) \\ 0.95 & (0.89-1.00)\end{array}$

Index persons with
high education
$(\mathrm{n}=121,902)$
IRR $\quad 95 \%$ CI

M-H test of homogeneity (p-value)
0.91
1.04
0.69
0.72
0.69
0.73
0.74
0.60
0.82

(0.88 - 0.94)

(0.95 - 1.14)

(0.70-0.92)

(0.50 - 0.93)

$(0.39-1.23)$

(0.53-0.89)

(0.67 - 0.78)

(0.61 - 0.88)

(0.52 - 0.69)

$(0.58-0.73)$

(0.74-0.92)

\section{Index persons with high education ( $n=121,902$ )} IRR $\quad 95 \% \mathrm{CI}$

\section{M-H test of homogeneity (p-value)}

\footnotetext{
All hospital admissions combined

Cancer [140-208 / C00-C97]

Respiratory diseases [460-519 / J00-J99]

Diabetes [250 / E10-E14]

Chronic liver disease [571 / K70,K73-K74]

Mental disorders [303,305 / F10]

Circulatory diseases [390-459 / 100-199]

- Cerebrovascular disease [430-438 / 160-169]

- Ischemic heart disease [410-414 / 120-125]

- Cerebrovascular or ischemic heart disease

- Other circulatory diseases
}

$\begin{array}{lll}(0.96-0.99) & 0.94 & (0.92-0.96) \\ (1.01-1.12) & 1.00 & (0.95-1.06) \\ (0.90-1.02) & 0.89 & (0.82-0.96) \\ (0.81-1.03) & 0.82 & (0.69-0.96) \\ (0.81-1.28) & 0.97 & (0.72-1.31) \\ (0.96-1.16) & 0.88 & (0.77-1.01) \\ (0.86-0.93) & 0.85 & (0.82-0.89) \\ (0.85-1.02) & 0.81 & (0.73-0.90) \\ (0.77-0.88) & 0.80 & (0.74-0.86) \\ (0.82-0.91) & 0.81 & (0.76-0.86) \\ (0.89-1.00) & 0.91 & (0.86-0.97)\end{array}$

0.02 *
0.12
0.14
0.27
0.78
0.03
0.11
0.04
0.48
0.10
0.44 *

Notes. The incidence rate ratio of hospital admissions corresponding to high versus low education of the mother [A], and the father $[B]$, separately for low and high educated index persons, along with the corresponding 95\% confidence intervals. Also, the $p$-value of the Mantel-Haenzel test for homogeneity between subgroups of individuals with low and high education is given. $I R R=$ incidence rate ratio; $C l=$ confidence interval; $M-H=$ Mantel-Haenzel; * $=p<0.05$ 
Tables 4A-B show the hazard rates from the Cox models for high versus low parental education level, separately for hospital admission and death. An interaction term for education of the mother and education of the father was initially included in the Cox model. However, this term was not significant in our analyses and subsequently dropped from the model.

\section{Hospital admission and death for low educated index persons}

From Table 4A we see that high maternal education level was associated with a $5 \%$ lower risk of hospital admission in general, but not with a lower risk of death for low educated individuals. Although the risk of hospital admission due to ischemic heart disease was lower, this was not the case for the risk of death due to ischaemic heart disease. Furthermore, the risk of hospital admission due to cerebrovascular disease was increased by $12 \%$, although not significantly, and the risk of death due to cerebrovascular disease was increased by $91 \%$ as well (significantly).

High paternal education level was significantly associated with a $7 \%$ increased risk of hospital admission due to cancer and a $15 \%$ increased risk of admission due to mental disorders, and with decreased hospital admission due to all types of circulatory diseases, most notably a $10 \%$ reduced risk of hospital admission due to ischaemic heart disease. Furthermore, high paternal education level was significantly associated with a $13 \%$ increased risk of death in general, specifically a $10 \%$ increased risk of death due to cancer and a $59 \%$ increased risk of death due to mental disorders.

Table 4. Effects of parental education level for index persons with low and high education, obtained from the Cox proportional hazard competing risk model, and adjusted for confounders.

[A]

Index persons with low education

$(n=337,064)$

All hospital admissions combined

Cancer [140-208 / C00-C97]

Respiratory diseases [460-519 / J00-J99]

Diabetes [250 / E10-E14]

Chronic liver disease [571 / K70,K73-K74]

Mental disorders [303,305 / F10]

Circulatory diseases [390-459 / 100-199]

- Cerebrovascular disease [430-438 / 160-169]

- Ischemic heart disease [410-414 / I20-125]

- Cerebrovascular or ischemic heart disease

- Other circulatory diseases

Above hospital admissions combined

Death due to all causes

Cancer [140-208 / C00-C97]

Respiratory diseases [460-519 / J00-J99]

Diabetes [250 / E10-E14]

Chronic liver disease [571 / K70,K73-K74]

Mental disorders [303,305 / F10]

Circulatory diseases [390-459 / 100-199]

- Cerebrovascular disease [430-438 / 160-169]

- Ischemic heart disease [410-414 / 120-125]

- Cerebrovascular or ischemic heart disease

- Other circulatory diseases

Above causes of death combined
High vs low education of the mother

Events HR

95\% Cl

159,769

19,911

0.95

13,712

0.99

3,912

1,093

5,987

37,986

7,409

15,667

22,204

15,782

69,826

12,453

6,215

438

268

388

287

0.84

0.91

0.72

0.98

0.92

1.12

0.80

0.89

0.96

0.92

1.12

1.01

0.52

0.83

1.43

1.57

2,695

1.21

620

1.91

1,446

0.78

2,066

629

1.10

1.53

10,291

$(0.90-0.99)$
$(0.86-1.14)$
$(0.70-1.02)$
$(0.65-1.28)$
$(0.35-1.45)$
$(0.76-1.26)$
$(0.83-1.03)$
$(0.89-1.41)$
$(0.67-0.97)$
$(0.77-1.03)$
$(0.82-1.14)$
$(0.85-0.99)$
$(0.94-1.33)$
$(0.79-1.31)$
$(0.13-2.13)$
$(0.46-1.50)$
$(0.62-3.28)$
$(0.63-3.92)$
$(0.83-1.76)$
$(1.01-3.64)$
$(0.41-1.46)$
$(0.70-1.73)$
$(0.78-3.01)$
$(0.87-1.29)$

1.00

(0.98 - 1.02)

(1.01 - 1.12)

(0.95 - 1.09)

(0.88 - 1.13)

(0.89 - 1.39)

(1.04 - 1.26)

(0.92 - 0.99)

(0.91 - 1.08)

(0.85 - 0.96)

(0.88 - 0.98)

(0.93 - 1.05)

(0.99 - 1.05)

(1.05 - 1.20)

(1.01 - 1.21)

(0.63 - 1.37)

(0.58 - 1.54)

(0.77 - 1.61)

(1.07 - 2.35)

(0.93 - 1.25)

(0.62 - 1.20)

(0.96 - 1.42)

(0.90 - 1.27)

(0.83 - 1.51)

(1.03 - 1.19) 
[B]

Index persons with high education

( $n=121,902$ )

All hospital admissions combined

Cancer [140-208 / C00-C97]

Respiratory diseases [460-519 / J00-J99]

Diabetes [250 / E10-E14]

Chronic liver disease [571 / K70,K73-K74]

Mental disorders [303,305 / F10]

Circulatory diseases [390-459 / 100-199]

- Cerebrovascular disease [430-438 / 160-169]

- Ischemic heart disease [410-414 / I20-125]

- Cerebrovascular or ischemic heart disease

- Other circulatory diseases

Above hospital admissions combined

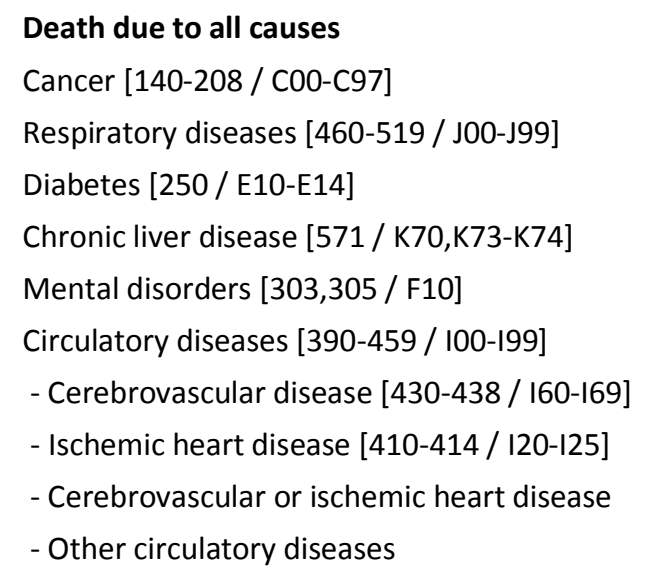

Above causes of death combined
High vs low education of the mother

Events 48,460

6,447

3,193

781

228

1,128

11,005

2,013

4,092

5,915

5,090

20,026

HR

ther

0.95

95\% Cl

(0.91 - 0.98)

(0.96 - 1.15)

(0.76 - 1.01)

(0.60 - 1.13)

(0.43 - 1.34)

(0.60 - 1.02)

(0.77 - 0.91)

(0.73 - 1.07)

(0.63-0.85)

(0.70 - 0.88)

(0.80 - 1.01)

(0.85 - 0.96)

0.90

2,782

0.97

(0.84 - 1.13)

0.97

(0.89 - 1.06)

1,565

0.96

(0.78 - 1.17)

0.89

(0.79 - 1.01)

(0.13 - 2.45)

0.87

(0.34 - 4.14)

0.91

(0.02 - 1.29)

1.49

(0.44 - 1.70)

(0.43 - 1.95)

(0.86 - 2.60)

(0.30 - 2.59)

1.75

(0.94 - 3.24)

(0.71 - 1.45)

0.90

$(0.73-1.11)$

$543 \quad 1.02$

(0.37 - 1.68)

1.11

(0.74 - 1.66)

(1.02 - 2.54)

0.76

(0.56 - 1.04)

(0.87 - 1.90)

0.87

(0.68 - 1.11)

(0.13 - 1.04)

0.99

(0.67 - 1.47)

(0.79 - 1.11)

0.92

Notes. Hazard rates and their 95\% confidence intervals for hospital admission or death due to selected disease categories, for subgroups of index persons with a low $[A]$ or high education level $[B]$, with parents with low or high education level. For each disease category the corresponding ICD 9 and ICD 10 codes are given, separated with a slash. Note that for hospital admissions the events in the separate disease categories do not add up to the events in 'combined' categories because patients may be admitted for multiple diseases sequentially and the 'combined' categories only account for the first admission of any patient.

\section{Hospital admission and death for high educated index persons}

Table 4B shows that a high level of education for either the mother or the father was associated with a $3-5 \%$ significantly decreased risk of hospital admission in general for index persons with high education. In particular, there was an association between high parental education and a reduced risk of hospital admission for circulatory diseases. However, this association was no longer observable for the corresponding risk of death. Moreover, high maternal education was significantly associated with a $61 \%$ increased risk of death due to ischaemic heart disease. Finally, high paternal education level was no longer significantly associated with an increased risk of admission or death due to mental disorders, as it was for index persons with low education.

\section{Cancer related hospital admission}

The hazard rates for hospital admission due to cancer, and the association with high education levels of the mother and father of the index persons, are shown in Tables 5A-B. Table 5A shows the results for low educated index persons and 
Table 5B shows the results for high educated index persons. In general, parental education level was not associated with substantially lower or higher risk of admission due to cancer. However, high paternal education level was associated with a marginally significant $5 \%$ increased risk of admission for any cancer type, and a $20 \%$ decreased risk of admission due to haematopoietic cancer for index persons with low education. Also, a high paternal education level may be linked to a $16 \%$ increased risk of admission for breast cancer in high educated women.

Table 5. Risk of hospital admission due to cancer related to parental education levels for index persons with low and high education, obtained from the Cox proportional hazard competing risk model, and adjusted for confounders

[A]

$$
\begin{aligned}
& \text { Index persons with low education } \\
& (n=337,064)
\end{aligned}
$$

\section{All admissions for cancer}

Cancer - digestive system [150-159]

Cancer - respiratory system [160-164]

Breast cancer [170]

Prostate cancer [177]

Kidney cancer [180]

Cancer - urinary organs [181]

Cancer - nervous system [193]

Hematopoietic cancer [200-209]

Other cancer types [194-199]

Above cancers combined

[B]

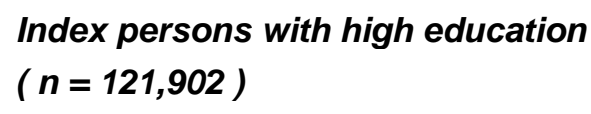

High vs low education of the mother

$\begin{array}{rl}\text { Events } & \boldsymbol{H R} \\ 25,437 & 1.06 \\ 3,821 & 1.07 \\ 2,090 & 0.99 \\ 5,481 & 1.22 \\ 3,070 & 1.00 \\ 582 & 0.18 \\ 860 & 0.58 \\ 850 & 0.70 \\ 1,550 & 1.47 \\ 1,547 & 1.08 \\ 19,355 & 1.04\end{array}$

High vs low education of the father

$\begin{array}{ccc}95 \% \mathbf{C l} & \boldsymbol{H} \boldsymbol{R} & \mathbf{9 5 \%} \boldsymbol{C l} \\ (0.94-1.20) & 1.05 & (1.00-1.10) \\ (0.77-1.48) & 0.97 & (0.85-1.09) \\ (0.63-1.55) & 1.06 & (0.90-1.24) \\ (0.96-1.55) & 1.06 & (0.97-1.17) \\ (0.69-1.46) & 1.13 & (0.99-1.29) \\ (0.03-1.32) & 0.97 & (0.71-1.34) \\ (0.24-1.40) & 1.21 & (0.95-1.54) \\ (0.31-1.57) & 1.11 & (0.87-1.42) \\ (0.94-2.31) & 0.80 & (0.65-0.98) \\ (0.66-1.76) & 1.13 & (0.94-1.35) \\ (0.91-1.20) & 1.04 & (0.99-1.10)\end{array}$

High vs low education of the mother

$\begin{array}{rlclc}\text { Events } & H R & 95 \% \mathrm{Cl} & H R & 95 \% \mathrm{Cl} \\ 8,609 & 0.99 & (0.91-1.07) & 1.03 & (0.98-1.08) \\ 1,189 & 1.03 & (0.83-1.29) & 1.01 & (0.88-1.15) \\ 392 & 0.89 & (0.59-1.36) & 0.93 & (0.73-1.19) \\ 1,668 & 0.98 & (0.83-1.16) & 1.16 & (1.04-1.29) \\ 1,757 & 0.96 & (0.79-1.17) & 1.00 & (0.90-1.12) \\ 160 & 0.94 & (0.49-1.79) & 0.98 & (0.67-1.42) \\ 271 & 0.96 & (0.59-1.56) & 1.06 & (0.80-1.41) \\ 329 & 1.29 & (0.89-1.89) & 1.02 & (0.79-1.32) \\ 585 & 1.05 & (0.77-1.42) & 1.03 & (0.85-1.25) \\ 445 & 0.63 & (0.42-0.97) & 1.06 & (0.85-1.32) \\ 6,609 & 0.98 & (0.89-1.07) & 1.04 & (0.99-1.11)\end{array}$

Notes. Hazard rates and their 95\% confidence intervals for hospital admission of death due to cancer, for subgroups of index persons with a low $[A]$ or high education level $[B]$, with parents with low or high education level. For each cancer type the corresponding ICD 7 codes are given. The risk of breast cancer was assessed for women only, whereas the risk of prostate cancer was assessed for men only. Note that for hospital admissions, the events in the separate categories do not add up to the events in the 'combined' category because patients may be admitted for multiple types of cancer sequentially and the combined category only accounts for the first admission of any patient. 


\section{Discussion}

\section{Addressing the research questions}

The main objective of the paper was to test the pathway hypothesis against the life course hypothesis. To reject the pathway hypothesis, it is sufficient to show that there is an independent effect of parental education on adult health status, not just an indirect effect through the individual's own educational attainments. In Table 4 we showed that there is an effect of parental education on hospitalization also after stratifying on own educational attainments. This result is shown for the group of low educated individuals in Table $4 \mathrm{~A}$ and for high educated individuals in Table 4B. For both groups, the results suggest that the effect mainly can be attributed to circulatory diseases. Our interpretation of these results is that we can reject the pathway hypothesis and maintain the life course hypothesis.

As described in the Introduction, the life course hypothesis can be further divided into (i) the accumulation, (ii) the critical period and (iii) social mobility hypotheses (Mishra et al., 2009). Under the accumulation hypothesis, the effect of staying in a particular SEP increases with the time spent in the SEP, while according to the critical period hypothesis, health or vulnerability to disease is to a large extent determined during a particular period of an individual's life course. Several previous studies have pointed out the very early period in one's life as of particular interest (Barker, 1995; Barker, 1999).

The support for the life course hypothesis obtained in this study: the independent effect of parental SEP on health later in life is in line with the critical period hypothesis, since parental educational attainments are closely associated with the individual's SEP during childhood and adolescence. To what extent the effect of SEP on health is accumulated over the life cycle is beyond the scope of this study and cannot be inferred from our results.

Under the social mobility hypothesis, the social mobility in itself, has an effect on health outcomes. The result that high paternal education is associated with increased mortality in low educated individuals, as shown in Table 4A, supports this hypothesis. Our interpretation of this result is that individuals originating from homes with high educated fathers who do not acquire more than compulsory schooling themselves, are on average adversely selected. They are more likely to have initial characteristics, or characteristics acquired early on, that are associated with both lower probability to obtain more than compulsory schooling and higher mortality, compared to the group originating from homes with low educated fathers. The estimated effect is thus not causal, but rather due to selection (Haas \& Fosse, 2008; Blumenshine et al., 2008; Jackson, 2009).

\section{Previous findings}

A systematic review of 29 previous studies on the relation between childhood SEP and health outcomes later in life has showed an independent relation between childhood SEP and the outcomes of mortality in stomach cancer and haemorrhagic stroke (Galobardes, Lynch \& Davey Smith, 2004). The reviewed studies also found a relation between childhood SEP together with SEP in adult life and mortality in coronary heart disease and lung cancer, as well as in respiratory-related deaths. An update of the review including 11 additional studies confirmed the inverse relation between childhood SEP and mortality for all but one of the reviewed studies (Galobardes, Lynch, \& Davey Smith, 2008). Adjusting for adult SEP accounted to a varying degree for the association.

An inverse effect of SEP measured in childhood and adolescence was found on lifestyle factors related to mortality in coronary heart disease, allcause mortality and cardiovascular mortality for a cohort of 49,323 Swedish males born 1949-1951 (Hemmingsson and Lundberg, 2005). The study found that a substantial part of socio-economic differences in cardiovascular decrease among middle-aged men can be attributed to SEP in early life. A persistent adverse effect of low childhood SEP on adult health was also found in a 1,000 cohort of 26 year old individuals born in New Zealand in 1972-1973, when investigating health outcomes, including dental health and substance abuse (Poulton et al., 2002). A long-lasting effect of parental SEP on several diseases, in particular mortality from cardiovascular disease, was found in a sample of about 5,500 men aged 35-64 years drawn from 27 workplaces in Scotland (Smith et al., 1997). Moreover, in a study on differences in mortality by cause of death between individuals originating from different SEP groups, an adverse effect of childhood SEP on mortality in stroke and stomach cancer in adulthood was found, when controlling for adult SEP (Smith et al., 1998). Finally, 
an excess all-cause mortality as well as mortality due to ischaemic heart disease was demonstrated in a cohort of Swedish men born in 1946-1955 for individuals with non-employed and manual labour parents (Vagero and Leon, 1994). These findings provide support for the cumulative life course hypothesis.

In contrast, support for the pathway hypothesis was found in a study on coronary heart disease incidence, chronic bronchitis and depression in a sample of about 10,000 UK civil servants aged 35-55 years (Marmot et al., 2001). Here, childhood SEP did not have an effect on adult health outcomes when controlling for the SEP of individual. A study on a sample of 2,636 Finnish men yielded similar results: individuals with low income were found to have an increased risk of all cause and cardiovascular mortality relative to high income men (Lynch et al., 1994). Finally, evidence from a schooling reform did not indicate a substantial, direct effect of parental education on child health (Lindeboom, Llena-Nozal, $\&$ van der Klaauw, 2009).

\section{Strengths}

In this study we used data on parental education, income and health outcomes for all individuals born in Sweden between 1940 and 1949. Our study extends several aspects of the previous literature, as our data allowed us to study actual endpoints for all diseases, rather than a specific subset of diseases occurring either later in life or during adulthood. Compared to other registrybased studies, our analyses are more comprehensive, as we used data on health outcomes for a general population, i.e. not restricted to any occupational group or group of workplaces, and were able to adjust for important and potentially confounding factors. Also, we studied the direct relation between health and education, rather than a relation based on social class or income. Finally, given our large cohort, we were able to effectively analyze health outcomes separately for children who move up and children who move down in educational levels. The sheer numbers allowed subgroup analyses for samples based on own educational attainments.

\section{Limitations}

Our study has certain limitations. First, we used the primary diagnosis codes for hospital admissions to detect hospital admission due to diseases. However, from our data it was not possible to distinguish admission for follow up examinations from admission due to the event itself. As a result, admissions found just after the age of 50 might have occurred for either a new first admission, e.g. for a myocardial infarction at the age of 51, or a follow up check after a myocardial infarction at the age of 49 . However, in both cases, the event of interest had occurred and only the time to event might have been recorded incorrectly. Moreover, this can only occur during the first 1 or 2 years of follow up. Second, we used income level measured at a single point in time as a proxy for SEP. Although such a proxy may be imprecise, we obtained the income of the individuals when they were $40-50$ years old, i.e. generally by the time their career would plateau and when their income should be relatively stable (Haider \& Solon, 2006). Third, we performed a large number of tests on our data, which may lead to chance findings. For example, high maternal education was associated with an $82 \%$ lower risk (not significant) of admission for kidney cancer for index persons with low education level, and high paternal education level was linked to a $16 \%$ statistically significant increased risk of admission for breast cancer for high educated women. These may be chance findings. Alternatively, the first finding may be explained by a study showing that conditions in utero, reflected by birth weight, may affect the risk of renal cell cancer in adulthood (Bergstrom, Lindblad, \& Wolk, 2001) whereas the second finding may be explained by a higher age at first childbirth and fewer children for high educated women, as well as a more stringent self-diagnosis and compliance with screening compared with low educated women (Heck and Pamuk, 1997; Hussain et al., 2008). Fourth, given our selection process, in which index persons with parents who died relatively young had to be excluded, the least healthy families may have been excluded from our analysis. This may have resulted in an underestimation of the effect of parental education on adult health. Hence, the risk of disease, and associated mortality, may be larger than indicated in our results for index persons with low versus high maternal or paternal education level. Finally, we assumed that within the period of our analysis, factors affecting illness behaviour and referral practices in Sweden did not change substantially. This may not hold for all diseases, but is more likely to hold for many of the severe and lifethreatening diseases on which we focus in our analysis. 


\section{Policy implications}

Our findings have implications for the evaluation of programmes aimed at improving education or reducing health inequalities, and for projections of future health care needs of populations. Improvements in population education levels may have health effects beyond the cohort attaining more education. Our results show that it may be misleading to use cross-sectional data to establish the relation between educational attainments and health when education is seen and used as marker of a life course disadvantage. At

least some of the health differences are acquired early on and educational attainments are correlated across generations. This, in turn, implies that the estimated health effects of educational policies designed to increase the educational level of the population are likely to be biased when the persistent effects of parental education are ignored. This bias should be added to the bias originating from unobserved confounders that may plague observational studies on the effects of educational attainments on various individual outcomes.

\section{Acknowledgements}

The research has been funded by The Swedish Council for Social Research (FAS) and The Institute for Evaluation of Labour Market and Education Policy (IFAU).

\section{References}

Barker, D. J. (1995). Fetal origins of coronary heart disease. British Medical Journal, 311(6998), 171-74. Retrieved from: http://www.ncbi.n/m.nih.gov/pubmed/7613432

Barlow, L., Westergren, K., Holmberg, L., \& Talback, M. (2009). The completeness of the Swedish Cancer Register: a sample survey for year 1998. Acta Oncologica, 48(1), 27-33. Retrieved from: http://www.ncbi.nlm.nih.gov/pubmed/18767000

Bergstrom, A., Lindblad, P., \& Wolk, A. (2001). Birth weight and risk of renal cell cancer. Kidney International, 59(3), 1110-1113. Retrieved from http://www.ncbi.nlm.nih.gov/pubmed/11231367

Blumenshine, S. L., Vann, W. F. Jr., Gizlice, Z., \& Lee, J. Y. (2008). Children's school performance: impact of general and oral health. Journal of public health dentistry, 68(2), 82-87. Retrieved from: http://www.ncbi.nlm.nih.gov/pubmed/18221320

Case, A., Lubotsky, D., \& Paxson, C. (2002). Economic status and health in childhood: The origins of the gradient. American Economic Review, 92(5), 1308-34. Retrieved from: http://apps.webofknowledge.com/InboundService.do?SID=S2G2il9AD4bnBKh8cLh\&product=WOS\&UT=00 $0180306100003 \&$ Init=Yes\&action=retrieve\&mode=FullRecord

Gakidou, E., Cowling, K., Lozano, R., \& Murray, C. J. (2010). Increased educational attainment and its effect on child mortality in 175 countries between 1970 and 2009: a systematic analysis. Lancet, 376(9745), 959-74. Retrieved from: http://www.ncbi.nlm.nih.gov/pubmed/20851260

Galobardes, B., Lynch, J. W., \& Davey Smith, G. (2008). Is the association between childhood socio-economic circumstances and cause-specific mortality established? Update of a systematic review. Journal of Epidemiology and Community Health, 62(5), 387-390. Retrieved from: http://www.ncbi.nlm.nih.gov/pubmed/18413449

Galobardes, B., Lynch, J. W., \& Davey Smith, G. (2004). Childhood socio-economic circumstances and cause-specific mortality in adulthood: systematic review and interpretation. Epidemiologic reviews, 26, 7-21. Retrieved from: http://www.ncbi.nlm.nih.gov/pubmed/15234944

Gluckman, P. D., Hanson, M. A., Cooper, C., \& Thornburg, K. L. (2008). Effect of in utero and early-life conditions on adult health and disease. The New England Journal of Medicine, (1), 61-73. Retrieved from: http://www.ncbi.nlm.nih.gov/pubmed/18596274

Haas, S. A., \& Fosse, N. E. (2008). Health and the educational attainment of adolescents: evidence from the NLSY97. Journal of Health and Social Behavior, 49(2), 178-92. Retrieved from: http://www.ncbi.nlm.nih.gov/pubmed/18649501

Haider, S., \& Solon, G. (2006). Life-cycle variation in the association between current and lifetime earnings. American Economic Review, 96(4), 1308-20. Retrieved from: http://apps.webofknowledge.com/InboundService.do?SID=S2G2il9AD4bnBKh8cLh\&product=WOS\&UT=00 $\underline{0240789200019 \& \text { Init=Yes\&action=retrieve\&mode=FullRecord }}$ 
Heck, K. E., \& Pamuk, E. R. (1997). Explaining the relation between education and postmenopausal breast cancer. American Journal of Epidemiology, 145(4), 366-72. Retrieved from: http://www.ncbi.nlm.nih.gov/pubmed/9054241

Hemmingsson, T., \& Lundberg, I. (2005). How far are socio-economic differences in coronary heart disease hospitalization, all-cause mortality and cardiovascular mortality among adult Swedish males attributable to negative childhood circumstances and behaviour in adolescence? International Journal of Epidemiology, 34(2), 260-267. Retrieved from: http://www.ncbi.nlm.nih.gov/pubmed/15333622

Hussain, S. K., Altieri, A., Sundquist, J., \& Hemminki, K. (2008). Influence of education level on breast cancer risk and survival in Sweden between 1990 and 2004. International Journal of Cancer, 122(1), 165-69. Retrieved from: http://www.ncbi.nlm.nih.gov/pubmed/17708572

Jackson, M. I. (2009). Understanding links between adolescent health and educational attainment. Demography, 46(4), 671-94. Retrieved from: http://www.ncbi.nlm.nih.gov/pubmed/20084824

Kirkwood, B. R. \& Sterne, J. A. C. (2003). Essential Medical Statistics (2nd ed.). York: Blackwell Science Ltd.

Kuh, D., Ben-Shlomo, Y., Lynch, J., Hallqvist, J., \& Power, C. (2003). Life course epidemiology. Journal of Epidemiology and Community Health, 57(10), 778-83. Retrieved from: http://www.ncbi.nlm.nih.gov/pubmed/14573579

Lindeboom, M., Llena-Nozal, A., \& van der Klaauw, B. (2009). Parental education and child health: evidence from a schooling reform. Journal of Health Economics, 28(1), 109-31. Retrieved from: http://www.ncbi.nlm.nih.gov/pubmed/18952306

Lynch, J. W., Kaplan, G. A., Cohen, R. D., Kauhanen, J., Wilson, T. W., Smith, N. L., \& Salonen, J. T. (1994). Childhood and adult socio-economic status as predictors of mortality in Finland. Lancet, 343(8896), 524-27. Retrieved from: http://www.ncbi.nlm.nih.gov/pubmed/7906766

Marmot, M., Shipley, M., Brunner, E., \& Hemingway, H. 2001. Relative contribution of early life and adult socioeconomic factors to adult morbidity in the Whitehall II study. Journal of Epidemiology and Community Health, 55(5), 301-7. Retrieved from: http://www.ncbi.nlm.nih.gov/pubmed/11297647

Mishra, G., Nitsch, D., Black, S., De Stavola, B., Kuh, D., \& Hardy, R. 2009. A structured approach to modelling the effects of binary exposure variables over the life course. International Journal of Epidemiology, 38(2), 52837. Retrieved from: http://www.ncbi.nlm.nih.gov/pubmed/19028777

Poulton, R., Caspi, A., Milne, B. J., Thomson, W. M., Taylor, A., Sears, M. R., \& Moffitt, T. E. (2002). Association between children's experience of socio-economic disadvantage and adult health: a life-course study. Lancet, 360(9346), 1640-1645. Retrieved from: http://www.ncbi.nlm.nih.gov/pubmed/12457787

Smith, G. D., Hart, C., Blane, D., Gillis, C., \& Hawthorne, V. (1997). Lifetime socio-economic position and mortality: prospective observational study. British Medical Journal, 314(7080), 547-52. Retrieved from: http://www.ncbi.nlm.nih.gov/pubmed/9055712

Smith, G. D., Hart, C., Blane, D., \& Hole, D. (1998). Adverse socio-economic conditions in childhood and cause specific adult mortality: prospective observational study. British Medical Journal, 316(7145), 631-35. Retrieved from: http://www.ncbi.nlm.nih.gov/pubmed/9603744

Socialstyrelsen. (2009)a. The Swedish National Patient Register. Inpatient diseases in Sweden 1987-2007. Stockholm, Sweden,. Retrieved from: http://www.socialstyrelsen.se/register/halsodataregister/patientregistret/inenglish

Socialstyrelsen. (2009b). The Swedish Cause of Death Registry. Causes of death. Stockholm, Sweden. Retrieved from: http://www.socialstyrelsen.se/register/dodsorsaksregistret

Socialstyrelsen. (2009)c. The Swedish Cancer Registry. Cancer incidence in Sweden. Stockholm, Sweden. Retrieved from: http://www.socialstyrelsen.se/register/halsodataregister/cancerregistret/inenglish

Statistics Sweden. (2004). The Swedish Register of Education. Stockholm, Sweden.

Retrieved from: http://www.scb.se/statistik/UF/UF0506/Produktbeskrivning short English UF0506 20040101r.doc

Statistics Sweden. (2009a). Population and Housing Census 1970. Stockholm, Sweden. Retrieved from: http://www.scb.se/default 2154.aspx

Statistics Sweden. (2009b). The Swedish Multigeneration Register. Stockholm, Sweden. Retrieved from: http://www.scb.se/default 2154.aspx

Statistics Sweden. (2009c). Longitudinal integration database for health insurance and labour market studies. Stockholm, Sweden. Retrieved from: http://www.scb.se/Pages/List 257743.aspx

Vagero, D., \& Leon, D. (1994). Effect of social class in childhood and adulthood on adult mortality. Lancet, 343(8907), 1224-25. Retrieved from: http://www.ncbi.nlm.nih.gov/pubmed/7909885 